\title{
Les dispositifs d'activation de la politique suédoise de l'emploi dans une perspective historique
}

Swedish unemployment and "activation" policy in an historical prospect

\section{Cyril Coulet}

\section{(2) OpenEdition}

\section{Journals}

Édition électronique

URL : http://journals.openedition.org/travailemploi/2199

DOI : 10.4000/travailemploi.2199

ISSN : 1775-416X

Éditeur

DARES - Ministère du Travail

\section{Édition imprimée}

Date de publication : 30 décembre 2007

Pagination : 63-74

ISSN : 0224-4365

\section{Référence électronique}

Cyril Coulet, «Les dispositifs d'activation de la politique suédoise de l'emploi dans une perspective historique », Travail et Emploi [En ligne], 112 | octobre-décembre 2007, mis en ligne le 05 novembre 2010, consulté le 19 avril 2019. URL : http://journals.openedition.org/travailemploi/2199; DOI : 10.4000/travailemploi.2199 


\title{
Les dispositifs d'activation de la politique suédoise de l'emploi dans une perspective historique
}

\author{
Cyril Coulet $\left.{ }^{*}\right)$
}

L'article synthétise sur plus d'un siècle les principales évolutions de la politique suédoise de l'emploi, souvent mise en exergue dans les travaux récents sur les politiques communautaires. Une perspective historique des institutions aide à mieux comprendre les évolutions de cette politique jusqu'aux réformes actuelles de l'assurance chômage en Suède liées au changement de gouvernement survenu à l'automne 2006. Cette réforme annonce une modification importante du référentiel de la politique de l'emploi suédoise.

La politique de l'emploi suédoise est souvent retenue comme référence dans les comparaisons internationales pour deux raisons. D'une part, les comparaisons entre les politiques de l'emploi, établies sur le fondement de la notion de taux d'emploi, tendent à conclure à une efficacité supérieure de la politique de l'emploi en Suède par rapport à celles menées dans d'autres pays. Le taux d'emploi s'élevait ainsi en 2003 à $45 \%$ pour les $15-24$ ans, $83,5 \%$ pour les 25-54 ans et 69\% pour les 55-64 ans en Suède contre respectivement $29,8 \%, 79,3 \%$ et $36,8 \%$ pour la France(1). D'autre part l'intérêt porté à la politique de l'emploi suédoise tient à l'engouement dont les «dépenses actives» ont fait l'objet. Ces dernières ont en effet retenu l'attention de l'OCDE dès 1964 et ont été portées au pinacle au cours des années 1990. L'union européenne a également assuré leur promotion dans le rapport de la Commission européenne sur la politique de l'emploi publié en 1997. Enfin, la stratégie de Lisbonne pour la croissance, inspirée de l'expérience scandinave, assure également la promotion des dispositifs inspirés par les «dépenses actives» de l'emploi.

Il est toutefois malaisé de comparer l'efficacité des politiques publiques alors que leur environnement social, juridique ou macroéconomique diffère. L'attention dont les «dépenses actives» ont fait l'objet de la part des organisations internationales citées s'est dans les faits accompagnée d'une méconnaissance de la spécificité suédoise (BARBIER, 2000). Il semble nécessaire d'examiner le processus de structuration et de fonctionnement des institutions

(*) Doctorant en sciences politiques auprès de l'université Paris I-Panthéon-Sorbonne, cyril.coulet@gmail.com

(1) OECD factbook 2005. Les données sont disponibles sur le site internet de l'OCDE. en charge de l'emploi pour comprendre la politique suédoise de lutte contre le chômage. En outre, il convient de dépasser la distinction opérée entre les «dépenses actives » et les «dépenses passives» afin de saisir la politique suédoise de l'emploi. D'une part les dépenses passives permettent de faciliter l'adéquation de l'offre et de la demande de travail et constituent à ce titre un élément régulateur du marché de l'emploi (FreYsSINET, 2000). D'autre part la forte substitution de l'État aux partenaires sociaux pour le financement de l'assurance chômage dans la plupart des pays européens a de facto conduit les «dépenses passives» à devenir un des éléments de la politique de l'emploi. Cet article se propose ainsi d'examiner les évolutions de la politique de l'emploi suédoise en transcendant la dichotomie opérée entre les «dépenses actives» et les «dépenses passives». L'approche historique retenue permet en effet d'éclairer les enjeux que recouvrent les différentes réformes de la politique suédoise de l'emploi.

\section{Les conditions d'élaboration d'une politique de l'emploi}

L'élaboration d'une politique de l'emploi n'est possible qu'à deux conditions. D'une part, il est indispensable qu'un marché de l'emploi existe, ce qui requiert la mobilité géographique et sectorielle de la main d'œuvre. D'autre part, le chômage doit être identifié et institué comme phénomène social. Or la création d'un marché de l'emploi ainsi que la construction du concept de chômage n'ont été réalisées qu'au terme d'un long processus qui aboutit au cours du XIX $\mathrm{X}^{\mathrm{e}}$ siècle. En effet, les sociétés européennes préindustrielles, régies par les corporations et par la transmission héréditaire des charges mécon- 
naissaient la notion d'inactivité. La peste noire qui traverse l'Europe au milieu du XIV siècle avait servi de fondement à l'élaboration des règles encadrant l'activité des personnes valides. Le Statut de travailleurs promulgué par Édouard III d'Angleterre en 1349 constitue ainsi le premier document énonçant la problématique du travail sous l'Ancien Régime. Cette dernière condamnait l'inactivité des valides et interdisait la mobilité géographique de la main-d'œuvre afin d'enrayer la progression des rémunérations. Des dispositions similaires seront adoptées dans l'Europe entière au cours du XIVe siècle. En outre, l'ordonnance adoptée par Richard II d'Angleterre en 1388 introduit également la condamnation de la mobilité sectorielle en Europe. La triple interdiction de la mobilité géographique, de la mobilité sectorielle et de l'inactivité des valides rendait impossible l'existence d'une politique de l'emploi. L'individu était ainsi nécessairement inscrit dans un cadre de sociabilité primaire qui devait lui assurer les moyens de sa subsistance. L'appartenance à une famille et, au-delà, à une communauté territoriale assignait en effet à chaque individu une raison sociale. L'assistance publique ne couvrait par conséquent que les cas d'indigence résultant d'une incapacité physique ou psychique de travailler (CASTEL, 2005). Les seules personnes valides prises en charge par l'assistance publique étaient alors les «pauvres honteux», produits d'une mobilité sociale descendante. Ces déclassés sociaux restaient régis par la logique de l'honneur qui leur interdisait de pratiquer une activité manuelle. Ils constituaient de ce fait la seule catégorie d'inactifs valides légitimement susceptible d'être prise en charge par la collectivité.

Toutefois, l'inactivité des valides dépassait par son ampleur la seule catégorie des déclassés sociaux. En effet, les variations climatiques, les guerres et les épidémies étaient causes de bouleversements. Aussi, ces sociétés durent reconnaître la permanence de situations d'inactivité pour les personnes valides et en organiser la gestion. L'assistance accordée aux inactifs ne pouvait toutefois remettre en question l'obligation de travailler pesant sur l'ensemble des valides. La prise en charge des inactifs par l'assistance publique était donc conditionnée à une obligation formelle de travailler. La traduction de cette obligation en dispositifs publics était toutefois complexe. Les tentatives visant à instaurer des «ateliers de secours», préludes aux «chantiers nationaux » de 1848, ou des Workhouses se solderont par des échecs. En définitive, l'obligation formelle de travailler ne se traduira concrètement que par la répression du vagabondage. La reconnaissance d'un droit individuel à la mobilité géographique constituera ainsi le préalable à la conception d'une politique de l'emploi. Toutefois, la mobilité de la main d'œuvre devait s'accompagner d'un démantèlement des corporations et des jurandes afin de permettre l'émergence d'un marché du travail. La libre circulation des travailleurs et le démantèle- ment des corporations sont presque concomitantes en Suède puisque la première est acquise en 1847 et la seconde en 1846. Toutefois, le chef-d'œuvre continue à être requis jusqu'en 1864 pour certains emplois. À cette date, il devient ainsi possible d'évoquer l'existence d'un véritable marché de l'emploi en Suède. Les règles héritées de l'Ancien Régime se prolongeront néanmoins comme en atteste la persistance du travail forcé pour les chômeurs au sein des Arbertshus jusqu'en 1885. De même, les derniers règlements assimilant l'inactivité d'un valide à un délit ne seront abrogés en Suède qu'en 1926.

La création d'un marché de l'emploi servit toutefois de prélude à l'émergence de la «question sociale». En effet, la libéralisation des flux de maind'œuvre ne permit pas de générer l'équilibre attendu sur le marché de l'emploi. On assista au contraire au développement du paupérisme et à la permanence de situations d'inactivité pour les adultes valides. Le besoin se fit ainsi sentir de réaliser des études statistiques sur la population en âge de travailler afin de mieux saisir l'ampleur des problèmes à traiter. L'élaboration de la population active comme catégorie statistique constitue donc l'élément déterminant dans l'identification du chômage comme phénomène social. L'État suédois commande ainsi les premières études statistiques sur la population active à la Direction générale du commerce et de l'industrie en 1906. Les résultats de ces études seront publiés en 1911 de sorte que l'ensemble des conditions est réuni à cette date pour qu'une politique de l'emploi puisse être élaborée en Suède.

\section{L'institution des agences communales de l'emploi}

L'obligation de territorialisation des demandeurs d'assistance publique a conduit l'échelon communal à devenir le cadre de référence des politiques sociales. De fait, la première loi sur l'assistance publique (Fattigvårdslag) adoptée en Suède en 1871 autorisait les communes à prendre toutes les mesures nécessaires pour assister les chômeurs en complément des emplois offerts sur le budget de l'État (OlofsSON, 1996, p. 116). L'intervention des communes dans le traitement du chômage prit ainsi une importance marquée au cours des années 1890 en raison d'une conjoncture économique dégradée. La seule ville de Stockholm employait près de 1000 chômeurs pour ses travaux de construction au cours de cette décennie. Les communes furent donc pionnières dans la réflexion sur le traitement du chômage comme l'illustre l'instauration de la première commission d'enquête sur cette question par la ville de Stockholm en 1894. Les conclusions de cette commission appelèrent toutefois à 1'organisation d'une politique de l'emploi imbriquant le niveau national et le niveau local. La commission portait 
en effet l'accent sur la réalisation d'études statistiques, sur la mise en place d'un réseau d'agences de l'emploi ainsi que sur l'appréhension de la faisabilité d'une assurance chômage (EDLING, 2005 p. 3). La diversité de ces propositions témoigne de l'effort consenti par les experts réunis au sein de la commission en vue d'intégrer les expériences menées dans les pays étrangers. La réalisation des études statistiques constituait la proposition la moins controversée et fut à ce titre la première à connaître une traduction opérationnelle. Or les experts de la Direction générale du commerce et de l'industrie nouvellement investis de la réalisation des études statistiques sur la population active préconisèrent rapidement la création d'agences communales de l'emploi dont l'accès serait gratuit pour les chômeurs. En effet, la constitution d'un réseau d'agences de l'emploi avait pour objet de favoriser l'adéquation de l'offre et de la demande de travail au sein des bassins d'emplois communaux. Les agences communales devaient faire office d'intermédiaires pour favoriser la diffusion des informations relatives à l'emploi sans assurer le versement de prestations.

La solution préconisée était loin d'être novatrice puisque des agences communales avaient déjà vu le jour en Allemagne notamment à Strasbourg, Stuttgart, Francfort et Munich. La ville pionnière en la matière étant la ville allemande d'Esslingen qui avait déjà ouvert une telle agence en 1894. Le modèle germanique des agences communales de l'emploi reposait sur trois critères: un office communal dirigé par un conseil comprenant des représentants des employeurs et des travailleurs, la gratuité de l'accès aux services et l'insertion des agences dans un réseau régional ou national. La création en 1898 de l'association allemande des agences de l'emploi permit la diffusion de ce modèle qui fut rapidement importé dans les pays nordiques. Les premières agences communales de l'emploi virent ainsi le jour en Suède à Helsingborg et à Göteborg en 1902. Le mouvement ouvrier, représenté par le syndicat LO et le parti social-démocrate, n'était toutefois pas favorablement disposé à l'implantation du modèle allemand d'agences communales de l'emploi. Le second congrès ouvrier scandinave organisé à Copenhague en 1888 prônait en effet le soutien financier public à des bourses du travail organisées par les syndicats (LINDBOM, 1938, p. 55). Toutefois la neutralité des agences communales résultant de la composition bipartite de leurs organes directeurs permit d'obtenir le ralliement du mouvement ouvrier à cette nouvelle organisation. En outre, le Parlement suédois consacra l'existence de ces agences en autorisant le versement de dotations étatiques de fonctionnement en 1907. Il conditionna ce versement à trois critères qui recoupaient largement ceux préconisés par l'association allemande des agences de l'emploi. Il exigeait ainsi 1'existence d'un conseil paritaire, la neutralité en cas de conflits sociaux et la délivrance de toute informa- tion utile aux demandeurs d'emplois (RothsteIn, 1988 p. 9). La représentation paritaire du patronat et des syndicats au sein des instances dirigeantes de ces agences a permis à ces entités de constituer un laboratoire de la concertation sociale (COULET, 2005). Le réseau des agences locales se développa passant de 100 agences en 1914 à 166 agences en 1939. Toutefois, l'impulsion la plus importante donnée à la constitution de ce réseau résulta de la centralisation de la politique de l'emploi organisée au cours de la Seconde Guerre mondiale puisque le nombre d'agences s'élevait à 246 en 1945. La constitution de ce réseau d'agences communales a été consensuelle dans la mesure où elle répondait aux besoins des différents acteurs. Ainsi, le patronat et les syndicats y voyaient un moyen de contenir le chômage. Le développement des agences permettait également de soulager les finances communales en réduisant les coûts de l'assistance publique. Enfin, ces agences offraient à l'État la possibilité d'affiner sa connaissance du marché de l'emploi grâce aux informations collectées sur le nombre de chômeurs et leur situation sociale.

\section{La genèse laborieuse de l'assurance chômage}

Le chômage pose un problème fondamental à la société puisqu'il équivaut à énoncer qu'un individu ne peut subvenir à ses propres besoins en l'absence de protection sociale. À l'instar des autres pays européens, les débats ont oscillé en Suède entre l'assistance accordée en contrepartie d'un travail (Arbetslinjen) et l'allocation d'un soutien financier en raison de la situation sociale des chômeurs (Understödslinjen). Or l'obligation de travailler a prévalu dans le traitement du chômage en Suède dès le XIX ${ }^{\mathrm{e}}$ siècle. Les premières caisses d'assurance chômage suédoises ont ainsi été constituées par les syndicats pour protéger leurs propres membres contre le risque du chômage. Les syndicats professionnels se sont en effet développés sur les décombres des corporations afin d'assurer le rôle de protection des travailleurs qui était jusqu'ici dévolu à ces dernières. Les caisses d'assurance chômage trouvent leur origine dans les caisses instituées pour financer la mobilité des artisans (Reshjälpskassa). L'assemblée des typographes a ainsi institué le premier syndicat suédois en 1886 consécutivement à l'instauration d'un fonds professionnel pour financer la mobilité de ses membres en 1883. Le fonds d'aide à la mobilité devait permettre aux travailleurs de supporter les frais de déplacement induits par la spécialisation sectorielle des bassins d'emplois. Le lien existant entre l'emploi saisonnier et la mobilité géographique a toutefois conduit à affecter directement aux chômeurs les sommes collectées pour promouvoir la mobilité dès 1893 . La solidarité professionnelle a donc servi de fondement à la première caisse d'assurance chômage en Suède. 
L'esprit de solidarité était en outre teinté de pragmatisme. En effet, la constitution de cette réserve financière avait pour objectif de renforcer la position du syndicat en offrant un service susceptible de motiver les adhésions. De même, le fonds d'assurance chômage pouvait être mobilisé en vue de financer les salariés grévistes. La création de ces fonds d'assurance chômage constituait ainsi un élément fondamental du financement des conflits sociaux. Dès lors, l'exemple du syndicat des typographes se diffusa au sein du mouvement syndical suédois. Ainsi, la Suède dénombrait vingt-quatre fonds d'assurance chômage couvrant 349800 salariés en 1934 alors qu'elle ne comprenait que neuf fonds couvrant 65500 salariés en 1907 (EDELBAKE, 1975, p. 13).

Les autorités politiques suédoises acceptèrent tardivement d'instaurer une assurance chômage en dépit de la multiplication des systèmes d'assurance à l'étranger. Ainsi, la première proposition de loi visant à instaurer une assurance chômage a été présentée en 1922 par le comité d'enquête sur l'assurance sociale. La proposition avait pour objet de subventionner les fonds privés détenus par les syndicats selon la logique du «système de Gand»(2). Ce modèle fut extrêmement populaire en Europe au début du siècle et fut adopté par différents États dont le Danemark en 1907. Toutefois, le système de Gand perdit de sa capacité d'attraction avec la création du modèle britannique d'assurance universelle en 1911 et du modèle allemand d'assurance chômage géré paritairement par les partenaires sociaux en 1927. En ce sens, l'adoption de l'ordonnance du 15 juin 1934 instituant une assurance chômage en Suède, sur les fondements du système de Gand apparaît anachronique au regard des expériences menées à l'étranger. En outre, cette décision semble paradoxale au regard de la défiance affichée par les pouvoirs publics suédois à l'égard de l'utilisation des fonds d'assurance chômage par les syndicats. L'importation du modèle britannique ou du modèle allemand d'assurance chômage aurait ainsi permis de contourner la gestion des fonds d'assurance chômage par les syndicats. Toutefois, ces expériences ont été systématiquement repoussées en raison du nombre de personnes assurées et du coût attendu de ces systèmes. En effet, le système britannique assurait 14 millions d'individus en 1934 et le système allemand 13,47 millions alors que le système suédois n'assurait que 350000 personnes et en indemnisait 41000 au cours de cette même

(2) Ce système fait référence à la ville du même nom qui établît en 1901 un système de subvention directe aux membres des syndicats inscrits dans un fonds d'assurance chômage. La municipalité complétait par ce biais l'allocation versée aux chômeurs résidant sur son territoire par les fonds d'assurance privés. Ainsi, le système de Gand désigne spécifiquement les systèmes assurantiels privés, reposant sur une cotisation volontaire des membres et subventionnés par des acteurs publics. année (3). De plus, la forte dégradation de la conjoncture économique en Suède au cours des années 1930 faisait craindre une explosion des dépenses publiques en cas d'extension de l'assurance chômage au-delà des caisses gérées par les syndicats. La victoire des sociaux-démocrates aux élections législatives de 1932 et l'accord passé le 27 mai 1933 avec le parti agrarien (Kohandeln) permirent de trouver un compromis permettant d'instituer une assurance chômage en Suède. L'assurance instituée était offerte à toute personne âgée de plus de 16 ans, capable de travailler et libre de tout engagement. Les individus devaient toutefois avoir cotisé à un fonds d'assurance chômage pendant cinquante-deux semaines avant de percevoir l'allocation-chômage. De même, ils devaient avoir travaillé pendant vingtsix semaines au cours des douze mois précédant la période de chômage. Enfin, le bénéficiaire de l'allocation-chômage devait se rendre quotidiennement auprès de son agence communale de l'emploi pour percevoir son allocation.

\section{Du conflit au consensus: I'institutionnalisation progressive de la politique de l'emploi}

Le versement d'une dotation de fonctionnement aux agences communales de l'emploi en 1907 consacre l'émergence de l'État comme acteur de la politique de l'emploi. Toutefois, la première autorité consultative nationale responsable de la lutte contre le chômage n'est apparue qu'au cours de la première guerre mondiale. Les nécessités de la mobilisation des ressources dans le contexte d'une guerre totale ont en effet présidé à la création de la commission nationale sur le chômage (Statens Arbetslöshetskommission, AK) en 1914. Cette commission disposait en outre de relais au sein des régions et des communes (Arbetslöshets Och Hjälpskommittén) en charge de l'examen de la situation des chômeurs. Elle put ainsi encourager l'activité des chômeurs par cet intermédiaire en proposant des emplois financés par les fonds publics ainsi que des formations professionnelles. Cette commission était initialement conçue comme une institution temporaire destinée à être supprimée à la fin de la guerre. Sa mission fut toutefois prolongée en raison d'une conjoncture économique dégradée. Elle joua ainsi un rôle important entre 1921 et 1924 en contribuant à financer la politique de grands travaux destinée à fournir des emplois aux chômeurs. Elle commença à affirmer à cette occasion son autorité sur les agences

(3) Cf. Social SECURITY BoARd, 1937. Cet ouvrage reprend les grandes lignes du rapport rédigé en 1935 par Bryce M. Stewart, Merrill G. Murray, W. R. Williamson \& Fred Jahn intitulé Unemployment Compensation, dans le cadre du President's Committee on Economic Security. 
municipales de l'emploi au travers des comités régionaux du chômage (Arbetslöshetskomittéer). Toutefois, la pensée économique de la commission était fortement influencée par le paradigme libéral qui estimait que le chômage était la seule résultante du coût trop élevé de la main-d'œuvre.

La commission encouragea ainsi les chômeurs à accepter des postes rémunérés en dessous du salaire moyen enregistré dans les régions concernées. De même, elle contraignait les chômeurs dépendant de son assistance à remplacer les salariés grévistes. Ces positions finirent par heurter le parti social-démocrate qui était organiquement lié aux formations syndicales. Dès la fin des années 1920, les municipalités détenues par les sociaux-démocrates initièrent une politique de l'emploi parallèle, indépendante de la commission nationale du chômage. Ces municipalités utilisèrent en effet les possibilités offertes par la loi pour offrir des emplois subventionnés rémunérés au prix du marché. L'accession des sociaux-démocrates au pouvoir dans un contexte de forte hausse du chômage renforça d'autant plus cette opposition que les municipalités «dissidentes » disposaient d'un soutien financier étatique. Toutefois, la commission nationale sur le chômage était protégée par le parti agrarien qui était alors le partenaire gouvernemental des sociaux-démocrates. Ainsi, la politique de l'emploi suédoise fit l'objet d'un affrontement entre deux paradigmes alors que le chômage augmentait fortement au cours des années 1930. La querelle politique portait ainsi sur le niveau des rémunérations à offrir aux chômeurs ainsi que sur l'utilisation des chômeurs comme des briseurs de grève. Toutefois, la centralité du travail dans l'assistance fournie aux chômeurs n'était contestée par aucune des deux parties comme en atteste la création de l'assurance chômage volontaire. En outre, l'adoption des lois sur les conventions collectives et la création des tribunaux du travail en 1928 marquèrent le début de l'apaisement dans les tensions sociales. L'amélioration sensible de la situation de l'emploi à partir de 1936 (cf. tableau $\mathrm{n}^{\mathrm{o}} 1$ ) contribua également à pacifier le débat sur la politique de l'emploi.

Tableau 1

Évolution du nombre des chômeurs en Suède au cours de la période 1927-1938

\begin{tabular}{|r|r|r|r|}
\hline $\mathbf{1 9 2 7}$ & 19200 & $\mathbf{1 9 3 3}$ & 164100 \\
\hline $\mathbf{1 9 2 8}$ & 16700 & $\mathbf{1 9 3 4}$ & 114800 \\
\hline $\mathbf{1 9 2 9}$ & 10200 & $\mathbf{1 9 3 5}$ & 61600 \\
\hline $\mathbf{1 9 3 0}$ & 13700 & $\mathbf{1 9 3 6}$ & 35600 \\
\hline $\mathbf{1 9 3 1}$ & 46500 & $\mathbf{1 9 3 7}$ & 18200 \\
\hline $\mathbf{1 9 3 2}$ & 113900 & $\mathbf{1 9 3 8}$ & 16200 \\
\hline
\end{tabular}

Source: Edebalk P.G., "Arbetslöshetensförsäkringsdebatten”. En studie I svensk socialpolitik 1892-1934, p. 129.

La substitution de la commission nationale du travail (Statens Arbetsmarknadskommission, AMK) à la précédente commission en 1939 constitua un tournant dans la politique de l'emploi suédoise. Bien que censée avoir un rôle plus réduit que la précédente commission, son rôle fut dans les faits beaucoup plus important. La «politique de neutralité» mise en œuvre pendant la Seconde Guerre mondiale supposait l'autosuffisance nationale. L'AMK procéda à l'identification des besoins en main-d'œuvre et à l'orientation de celle-ci en vue de garantir le meilleur fonctionnement possible de l'économie. Cette réflexion aboutit à l'organisation de formation professionnelle en vue de pourvoir aux besoins de l'industrie dès mai 1940 (Sou (4), 1944). De même, les agences de l'emploi furent mobilisées en vue de procéder à l'orientation professionnelle des chômeurs. Enfin, l'État augmenta très sensiblement ses aides à la mobilité géographique. Les expériences menées au cours de la guerre furent reprises par la commission Myrdal sur la planification économique en 1944. Celle-ci préconisait la rationalisation de l'économie par l'amélioration de la mobilité du travail. Ainsi, l'État devait veiller à améliorer le placement des demandeurs d'emploi par les agences communales de l'emploi. Ces dernières devaient notamment veiller à organiser l'orientation professionnelle des chômeurs. Enfin, l'État devait encourager la mobilité de la main-d'œuvre en offrant des aides à la mobilité géographique ainsi que des aides pour la formation professionnelle.

Toutefois, les préconisations de la commission Myrdal restèrent lettre morte en raison de l'amélioration de la situation de l'emploi en Suède dans l'immédiat après-guerre. En effet, la politique de l'emploi restait conçue comme une politique contracyclique dont la pertinence diminuait en raison de la forte croissance économique suédoise soutenue par la demande internationale. Néanmoins, la réduction des moyens consacrés à la politique de l'emploi ne remit pas en question le consensus existant sur l'utilité d'une autorité nationale de l'emploi en Suède. Ainsi, l'octroi à l'AMK du contrôle de l'ensemble des subventions octroyées aux organes en charge de la lutte contre le chômage constitue en 1946 le dernier temps du processus de centralisation. Le remplacement de l'AMK par l'actuelle direction nationale du travail (Arbetsmarknadsstyrelsen, AMS) en 1948 constitue ainsi la dernière étape de consolidation du dispositif institutionnel suédois en matière d'emploi.

\section{L'affirmation des dépenses actives de l'emploi au sein du modèle Rehn-Meidner}

L'existence d'une autorité nationale de l'emploi a été fortement liée à l'affirmation d'une politique

(4) Statens offentliga utredningar, rapports produits par les commissions d'enquête parlementaire. 
active de l'emploi en Suède. Ainsi le rapport qui a présidé à la création d'AMS soulignait que la commission ne devait pas: "se contenter d'attendre le développement sur le marché de l'emploi mais devait chercher à le prévenir et - quand cela est possible - à l'influencer» (Sou, 1947). La politique active de l'emploi est toutefois associée au nom de deux économistes du syndicat LO: Gösta Rehn et Rudolf Meidner. Ces deux hommes ont en effet élaboré le modèle économique suédois dont les grandes lignes avaient été présentées dans le rapport intitulé: «Le mouvement syndical et le plein-emploi» lors du congrès de la confédération LO en 1951. Le programme d'action reposait sur les points suivants: une politique active sur le marché de l'emploi associée à des aides sélectives à l'embauche, une politique de détermination équitable des salaires et une politique fiscale restrictive. La combinaison de ces différents facteurs avait pour objectif d'atteindre le plein-emploi sans générer d'inflation. La politique active de l'emploi constituait ainsi un élément central du modèle Rehn-Meidner qui fut agréé par les autorités suédoises à l'issue d'une conférence réunissant le gouvernement et la direction du syndicat LO en 1955. Cette politique concernait principalement l'offre de travail puisque ces composantes étaient la formation professionnelle, l'orientation professionnelle et les aides à la mobilité géographique. La demande de travail était plus marginalement soutenue au travers des aides à l'embauche distribuées aux entreprises qui réalisaient des investissements ou qui procédaient à des recrutements. Le choix de soutenir les entreprises les plus dynamiques répondait à l'ambition d'accompagner la restructuration de l'économie suédoise. La politique active de l'emploi constituait à cet égard un des éléments permettant d'accélérer les mutations au sein de l'économie en fournissant de la main-d'œuvre aux secteurs les plus dynamiques. Cette politique était complétée par la recherche de l'équité dans la rémunération du facteur travail au sein des négociations salariales centralisées. Le principe d'une rémunération égale pour un emploi égal conduisait les entreprises les moins rentables à se moderniser sous peine de disparaître. La main d'œuvre ainsi libérée pouvait être réorientée vers les secteurs les plus dynamiques. La politique de l'emploi contenue dans le modèle Rehn-Meidner était ainsi très novatrice puisqu'elle aspirait à accélérer la fluidité du marché du travail pour accompagner la mutation industrielle de la Suède.

L'application de cette politique de l'emploi fit assez rapidement l'objet d'un consensus au sein de la classe politique et des partenaires sociaux dans la mesure où les objectifs en terme de croissance et de plein-emploi étaient atteints. Toutefois, la remise en cause du modèle Rehn-Meidner intervint avec les deux chocs d'offre induits par la crise pétrolière ainsi que par la fin du système de Bretton Woods. De plus, la concurrence du Japon et des nouveaux pays industrialisés avait induit une perte de parts de marché des entreprises suédoises à l'international. Les gouvernements suédois répondirent à cette nouvelle situation par un recours systématique à la dévaluation en vue de restaurer la compétitivité des entreprises suédoises. Ces dévaluations eurent pour conséquence de provoquer des chocs de demande positifs alors que les gouvernements menaient des politiques macroéconomiques expansives. L'inflation sous-jacente progressa graduellement, ce qui allait poser ultérieurement la question de l'arbitrage entre le chômage et l'inflation conformément à la courbe de Philips. De fait, la politique de l'emploi suédoise changea graduellement de paradigme pour devenir plus défensive. L'accompagnement des restructurations ne portait plus sur les secteurs en expansion mais sur les secteurs concurrencés par les nouveaux acteurs économiques asiatiques. Des aides massives furent notamment consenties aux entreprises sidérurgiques en vue de faciliter leur adaptation à la nouvelle concurrence internationale. En outre, la politique de l'emploi s'appuya sur le secteur public pour faire progresser le taux d'emploi. Ainsi, la part de l'emploi public passa de 25,8 à 38,2\% de l'emploi total entre 1970 et 1985 . L'expansion du secteur public s'inscrivait également dans le cadre de la loi sur l'interdiction des discriminations entre les sexes (Lag Om Förbud Mot Könsdikriminering) adoptée en 1980(5). En effet, l'objectif de promotion de l'emploi féminin s'est d'abord traduit par une forte féminisation des effectifs du secteur public. Enfin, le système des négociations salariales centralisées fut remis en cause par le retrait de l'association des employeurs de la métallurgie en 1983. Ainsi, la politique d'équité salariale qui était le complément de la composante active de la politique de l'emploi dans le modèle Rehn-Meidner disparut également. Le modèle Rehn-Meidner fragilisé au cours des années 1970 a perdu sa cohérence au cours des années 1980. Ces changements ont ainsi affecté la politique de l'emploi dont le caractère défensif de la politique de l'emploi suédoise se retrouve également dans l'adoption de la loi sur la protection de l'emploi (Lag Om Anställningsskydd) en 1982.

\section{La redéfinition des composantes actives de la politique de l'emploi depuis les années 1990}

Le début des années 1990 fut caractérisé en Suède par une inversion des priorités dans l'arbi-

(5) Le présent article ne peut toutefois examiner dans le détail les dispositifs légaux spécifiques conçus pour les groupes souffrant de discriminations à l'embauche. Cette catégorie regroupe les trois publics suivants: les femmes, les étrangers et les handicapés. 
trage effectué entre chômage et inflation. La priorité accordée à la lutte contre l'inflation eut pour conséquence de placer la Suède dans la période de récession économique la plus grave depuis les années 1930. En effet, le PIB ne progressa sur l'ensemble de la période 1990-1995 que de 0,6\% en moyenne. En outre, la Suède connut trois années consécutives de récession économique entre 1991 et 1993. Le choc économique induit par la politique de la déflation fut proportionné à la situation de surchauffe dans laquelle se trouvait l'économie suédoise. Les répercussions en matière d'emploi furent par conséquent très importantes puisque le chômage passa de 1,7\% en 1990 à 9,4\% en 1994. En effet, le secteur public entama une réduction de ses effectifs pour diminuer les déficits publics qui conduit à une diminution du taux d'emploi de près de six points au cours de la même période.

Tableau 2

Évolution comparée du taux de chômage en Suède et dans l'Union européenne de 1990 à 2004

\begin{tabular}{|c|c|c|}
\hline & Suède & $\begin{array}{c}\text { Union européenne } \\
\text { à quinze }\end{array}$ \\
\hline $\mathbf{1 9 9 0}$ & 1,7 & 8,1 \\
\hline $\mathbf{1 9 9 4}$ & 9,4 & 10,5 \\
\hline $\mathbf{1 9 9 9}$ & 6,7 & 8,7 \\
\hline $\mathbf{2 0 0 3}$ & 5,6 & 8,0 \\
\hline $\mathbf{2 0 0 4}$ & 6,4 & 8,0 \\
\hline
\end{tabular}

Source: OECD, Employment Outlook 2005, Statistical Annex, Table B.

Tableau 3

Évolution comparée du taux d'emploi en Suède et dans l'Union européenne de 1990 à 2004

\begin{tabular}{|c|c|c|}
\hline & Suède & $\begin{array}{c}\text { Union européenne } \\
\text { à quinze }\end{array}$ \\
\hline $\mathbf{1 9 9 0}$ & 84,6 & 67,1 \\
\hline $\mathbf{1 9 9 9}$ & 78,5 & 69,0 \\
\hline $\mathbf{2 0 0 3}$ & 78,9 & 70,3 \\
\hline $\mathbf{2 0 0 4}$ & 78,7 & 70,8 \\
\hline
\end{tabular}

Source: OECD, Employment Outlook 2005, Statistical Annex, Table B.

Le retour du chômage en Suède a servi de cadre à la redéfinition des moyens et des finalités de la composante active au sein de la politique de l'emploi suédoise. En effet, les finalités assignées aux dépenses actives dans le cadre du modèle RehnMeidner devaient être reconsidérées consécutivement au bouleversement de l'environnement macroéconomique suédois. La composante active a ainsi reçu deux finalités au sein de la politique de l'emploi dans les années 1990. D’une part, elle continue à être l'instrument d'amélioration du marché de l'emploi en réduisant le taux de vacance des postes en agissant sur l'offre d'emploi. Toutefois, il n'est plus explicitement mentionné qu'elle doit être l'instrument de la restructuration des activités productives. D'autre part, elle permet d'exercer une pression sur certaines caté- gories de chômeurs en conditionnant le versement des indemnités à leur participation à des programmes spécifiques. Or la politique de l'emploi avait pour ambition d'atteindre le plein-emploi en permettant à chacun de choisir son emploi en toute liberté dans le modèle Rehn-Meidner. Dans les années 1990, l'accent fut porté sur la nécessité d'élever le taux d'activité pour financer les politiques sociales sans compromettre la situation des finances publiques. Ainsi, la notion de liberté dans le choix de l'emploi a disparu des objectifs de la politique de l'emploi suédoise. En outre, la persistance d'un taux de chômage autour de $6 \%$ a conduit le gouvernement à ne plus faire de la recherche du plein-emploi le seul objectif de la politique de l'emploi. Les programmes de la composante active de l'emploi se sont donc vus assigner pour but de maintenir ou de développer les compétences des chômeurs à défaut de leur offrir un emploi.

De nouveaux programmes relatifs à la composante active de la politique de l'emploi furent ainsi initiés dans les années 1990. Le premier d'entre eux est le programme municipal pour les jeunes (Kommunala Ungdomsprogrammet) lancé en 1995. Ce programme destiné au moins de 20 ans a été complété par le programme "garantie d'activité pour les jeunes» (Ungdomsgarantin) initié en 1998, qui a pour cible les jeunes âgés de 20 à 24 ans. Ces deux programmes présentent deux caractéristiques qui les distinguent des précédents dispositifs de la composante active de l'emploi. D'une part, ils dépassent par leur ampleur les premiers programmes pour les jeunes instaurés en 1984. Ils combinent en effet la formation professionnelle et les expériences professionnelles tout en garantissant aux personnes concernées un revenu suffisant afin de construire un projet professionnel. D'autre part, ces programmes sont pilotés par les communes alors que le rôle de ces dernières était jusqu'ici limité au financement des dépenses sociales pour les chômeurs en fin de droit. Ainsi, les autorités nationales de l'emploi ont perdu le monopole qu'elles détenaient jusqu'ici dans la gestion de la composante active de la politique de l'emploi. Cette évolution a été encouragée par le gouvernement qui a incité les communes à s'impliquer dans la lutte contre le chômage de longue durée afin de rapprocher l'aide sociale de la politique de l'emploi conformément aux expériences menées au Danemark au cours des années 1990 (FinANSDEPartementet, 2006, p. 30). De fait, $70 \%$ des communes suédoises disposaient d'un département en charge des questions relatives au marché du travail en 1998 contre seulement $20 \%$ en 1995. L'activisme communal, souhaité par l'État, a induit une véritable décentralisation de la politique de l'emploi en Suède (Sou, 2000). Toutefois, cette évolution a quelque peu altéré la logique initiale de la politique active de l'emploi en réduisant la mobilité géographique des personnes prises en charge par les autorités municipales (HJERTNER THORÉN, 2005). Par ailleurs, la politique de l'emploi 
suédoise a renoué avec l'ambition d'accompagner les mutations industrielles de l'économie au cours des années 1990. Ainsi, les programmes Swit ou datortek ont porté sur l'adaptation des compétences des chômeurs aux besoins des secteurs de pointe. Toutefois, le programme phare de la composante active de la politique de l'emploi est la garantie d'activité (Aktivitetsgarantin) instaurée en 2000. Ce programme est remarquable à deux titres: d'une part, il n'est pas limité dans le temps contrairement aux autres programmes qui ne peuvent excéder six mois. D'autre part, il se situe à la frontière de la politique de l'emploi et de l'aide sociale puisqu'il concerne les chômeurs de longue durée inscrits depuis plus de vingt-quatre mois auprès d'une agence communale de l'emploi. Ces derniers sont alors sur le point de sortir du dispositif d'assurance chômage pour entrer dans le dispositif d'assistance publique. La garantie d'activité regroupait ainsi 45000 demandeurs d'emploi en 2005 dont le profil médian est celui d'un homme de 46 ans. Enfin, l'un des objectifs de ce programme est d'assurer un contrôle plus important des demandeurs d'emploi en imposant des contacts réguliers avec un agent responsable. Qui plus est, les demandeurs d'emploi sont dans l'obligation de définir un projet professionnel en contrepartie de l'accès à des formations leur permettant de maintenir ou d'améliorer leur employabilité.

L'évolution la plus remarquable de la politique active de l'emploi concerne toutefois les dépenses passives qui ont été progressivement mobilisées pour assurer le contrôle des demandeurs d'emploi. En ce sens, certains auteurs ont évoqué l'instauration d'un Workfare nordique dont les modalités diffèrent toutefois par rapport aux pays anglo-saxons (KvIST, 2003). Cette évolution a concerné autant la composante active que la composante passive de la politique suédoise de l'emploi selon un processus similaire à ce qu'a connu le Danemark. Les similitudes entre les réformes du marché du travail menées dans ces deux pays sont logiques au regard du rôle central tenu par les partis sociaux-démocrates. En effet, une trame commune à l'ensemble des mouvements sociaux démocrates a pu être définie pour la réforme des marchés de l'emploi au sein du SAMAK(6). La volonté d'activer les dépenses passives s'est d'abord traduite par une diminution des allocations chômage qui ont été portées de $90 \%$ à $80 \%$ du salaire brut en 1993 et à $75 \%$ du salaire brut entre 1996 et 1997.

(6) SAMAK, "Ett nordikst handlingsprogram för full sysselsättning », Stockholm, 2000. SAMAK est l'abréviation utilisée pour désigner un groupe de réflexion commun aux partis sociaux démocrates nordiques dont l'appellation complète est: Arbetarrörelsens nordiska samarbetskommité (groupe de travail commun aux mouvements nordiques de travailleurs). Ce groupe a fortement contribué à définir les caractéristiques $\mathrm{du}$ «modèle nordique» afin d'en assurer la promotion au sein des organisations internationales dont les pays nordiques sont membres.
En outre, la période de cotisation ouvrant droit à l'allocation-chômage a été graduellement relevée. Cette démarche a été prolongée par un contrôle accru exercé sur les demandeurs d'emploi avec l'instauration en 2000 d'un plan d'action individuel. Ce document sert à définir la trajectoire de retour à l'emploi du chômeur en cernant le secteur d'activité et la zone géographique concernés par sa recherche. Il contient également une énumération des droits et surtout des obligations du demandeur d'emploi qui permet l'instauration d'une relation contractuelle avec le service public de l'emploi. L'objectif ultime de cette démarche contractuelle est de responsabiliser le demandeur d'emploi afin de le rendre pleinement acteur de sa recherche. Cette démarche de responsabilisation a du reste été complétée par l'instauration en 2001 d'une dégressivité croissante des allocations d'assurance chômage en cas de refus d'un «emploi convenable» (Lämpligt Arbete) ou en cas de refus de participer à un programme de formation. Les allocations peuvent en effet être réduites de $25 \%$ en cas de premier refus, de $50 \%$ en cas de deuxième refus et supprimées en cas de troisième refus. L'allocation-chômage n'est donc plus seulement un moyen d'assurer un revenu de remplacement au cours de la période d'inactivité mais elle devient un élément à part entière de la nouvelle stratégie d'activation de la politique de l'emploi. En outre, la période d'indemnisation a été limitée à trois cents jours ouvrables maximum pour le versement de 1'allocation-chômage en 2001(7). Cette limitation était toutefois tempérée par le fait qu'une prestation complémentaire (Aktivitetstöd) pouvait être versée pendant trois cents jours ouvrables aux personnes engagées dans des programmes de formation. La volonté de durcir les conditions d'octroi des prestations sociales liées à l'emploi se retrouve également dans la polémique autour des congés d'assurance-maladie qui ne semble pas avoir été close par la réforme du $1^{\mathrm{er}}$ janvier 2005.

L'accession au pouvoir de la coalition bourgeoise consécutivement aux élections législatives de septembre 2006 doit beaucoup à la thématique de l'emploi qui a été placée au cœur de la campagne par les Modérés. Aussi, l'une des premières décisions du nouveau gouvernement a été de faire voter en décembre 2006 une loi modifiant de manière importante le système d'assurance chômage suédois. L'allocation-chômage est désormais dégressive puisqu'elle s'élève à $80 \%$ du salaire moyen de référence sur les douze derniers mois pendant 200 jours. Elle représente $70 \%$ du salaire moyen jusqu'au $300^{\mathrm{e}}$ jour et $65 \%$ à compter du $301^{\mathrm{e}}$ jour. Cette réforme entrée en vigueur cette année s'inscrit dans la continuité des réformes adoptées en 2001 qui tendaient à

(7) Les allocations chômage sont calculées sur une base quotidienne. Les 300 jours n'intègrent donc pas les week-end, ce qui porte à 420 jours la durée maximale d'indemnisation. 
faire des allocations chômage un instrument actif de la politique de l'emploi. En outre, la durée maximale cumulée de versement de l'allocation-chômage et de l'allocation complémentaire ne peut excéder quatre cent cinquante jours (8). Ainsi, les réformes qui ont été adoptées ne marquent paradoxalement pas de rupture par rapport à l'esprit des précédentes mesures prises par les sociaux-démocrates en Suède. Elles accordent néanmoins un primat à la reprise immédiate d'un emploi par rapport aux mesures de formation conformément à l'orientation fixée par le gouvernement libéral au Danemark en 2002. Cette orientation se dessine également dans les mesures de dégrèvement fiscal adoptées pour stimuler l'emploi notamment dans les services à la personne.

\section{La remise en cause du système de Gand par l'affirmation de l'assurance chômage universelle}

Le modèle de l'assurance chômage hérité du système de Gand a également évolué au cours de ces dernières années. Ainsi, la loi de 1997 sur les caisses d'assurance chômage (Lagen Om Arbetslöshetskassor) a porté création d'une nouvelle caisse intitulée Alfa-Kassan indépendante des syndicats. La création de cette caisse répondait à une demande ancienne des Modérés qui avaient déjà soumis en 1990 une proposition de loi visant à instaurer une assurance chômage pour l'ensemble des actifs (SocIALFÖRSÄKRINGSUTREDNINGEN, 2005, p. 50). Alfa-Kassan peut ainsi assurer le bénéfice de la prestation de base (Grundförsäkring) à l'ensemble des actifs, y compris les indépendants. En effet, le bénéfice de l'assurance chômage était réservé aux seuls membres des organisations syndicales jusqu'en 1998. Toutefois, la population couverte était importante puisqu'elle représentait $80 \%$ des salariés, auxquels il convient d'ajouter les étudiants qui au cours de certaines périodes pouvaient bénéficier de l'assurance chômage. La réforme des caisses d'assurance chômage n'a en revanche pas remis en cause l'existence des trente-six associations responsables de la gestion des caisses au niveau de la branche. Toutefois, la loi sur les caisses d'assurance chômage a prévu la création d'une autorité chargée d'opérer un contrôle sur la gestion des caisses par les associations. Ainsi, la nouvelle inspection de l'assurance chômage (Inspektionen För Arbetslöshetsförsäkringen) est chargée depuis 2004 de superviser l'action des caisses d'assurance chômage en remplacement de la précédente autorité (AMS Försäkringsenhet) qui assurait ce

(8) Les indemnités restant basées sur une base quotidienne, la durée totale de versement des deux allocations ne peut en définitive excéder 630 jours. contrôle depuis 1948. En outre, la préservation de la structuration institutionnelle héritée du décret de 1934 a été contredite par l'évolution du mode de financement de l'assurance chômage. En effet, les cotisations volontaires des employés n'ont eu de cesse de diminuer dans le financement de l'assurance chômage comme en atteste le tableau $n^{\circ} 4$. En ce sens, il devenait difficile d'évoquer l'existence d'une assurance chômage alors que les cotisations ne représentaient plus que $5 \%$ du financement de ces caisses. De fait, l'État peut, en sa qualité de principal contributeur au régime d'assurance chômage, déterminer par la loi les conditions d'ouverture des droits ainsi que le montant des prestations d'assurance chômage. Aussi, l'État est le grand responsable de l'équilibre de l'assurance chômage même si les syndicats continuent de gérer les associations en charge des caisses.

Tableau 4

Évolution du taux de prise en charge par l'État des allocations chômage de 1935 à 2008 (en pourcentage)

\begin{tabular}{|c|c|c|c|}
\hline $\mathbf{1 9 3 5}$ & 35 & $\mathbf{1 9 8 5}$ & 95 \\
\hline $\mathbf{1 9 4 5}$ & 43 & $\mathbf{1 9 9 4}$ & 100 \\
\hline $\mathbf{1 9 5 5}$ & 52 & $\mathbf{1 9 9 5}$ & 96,6 \\
\hline $\mathbf{1 9 6 5}$ & 67 & $\mathbf{1 9 9 7}$ & 94,9 \\
\hline $\mathbf{1 9 7 5}$ & 77,3 & $\mathbf{2 0 0 7}$ & $55^{*}$ \\
\hline
\end{tabular}

Source: Statskontoret, Vem ser efter arbetlöshetskassorna? 1999, p. 23.

* Le pourcentage indiqué pour 2007 est une estimation tenant compte du transfert des charges à destination des caisses d'assurance chômage.

De fait, le gouvernement suédois actuel a impulsé une réforme importante du mode de financement de l'assurance chômage en imposant aux caisses d'assurance chômage de verser 10 milliards de couronnes suédoises supplémentaires par an à la caisse nationale. Cette décision implique une réduction de la participation de l'État à $55 \%$ environ du financement de l'assurance chômage qui contraint les caisses d'assurance chômage à augmenter d'autant les cotisations de leurs membres. En effet, la réforme témoigne de la volonté du gouvernement suédois actuel de faire évoluer l'assurance chômage vers une assurance universelle. En effet, les cotisations des salariés seront modulées en fonction du risque chômage pesant au sein de chaque branche. La montée en charge des cotisations individuelles dans le financement de l'assurance chômage fait ainsi écho à la logique de responsabilisation des assurés sociaux qui devront supporter une part plus importante du financement de l'assurance chômage. Enfin, le gouvernement a chargé une commission d'enquête d'examiner les modalités de constitution d'une assurance chômage obligatoire pour les prestations complémentaires liées au revenu (Inkom stbortfallsförsäkring) qui compléterait l'allocationchômage universelle de base (Grundförsäkring). En effet, seuls 3,6 millions de Suédois cotisent à une assurance chômage alors que la population active 
comprend 4,6 millions de personnes. La commission d'enquête est invitée à formuler des propositions en vue de présenter une loi au Parlement avant la fin de l'année 2008. L'adoption de cette loi parachèverait l'évolution de l'assurance chômage vers une individualisation plus marquée des droits et des prestations.
$*$

Les réformes entreprises à partir des années 1990 dans la politique de l'emploi en Suède comme au Danemark aspirent à favoriser un taux d'emploi élevé afin de financer l'État-providence. Elles sont parfaitement cohérentes avec le primat traditionnellement accordé au travail par rapport aux prestations sociales dans la politique suédoise de l'emploi. En outre, les spécificités du modèle d'activation des pays nordiques persistent avec l'importance des dispositifs de formation ainsi que des allocations chômage relativement généreuses. Ces caractéristiques distinguent l'activation (Aktivering) des pays nordiques des politiques de Workfare américaine ou de Welfare to Work britannique. Toutefois, les réformes adoptées en 2006 introduisent des inflexions importantes de la politique suédoise de l'emploi qui préfigurent des évolutions possibles du Welfare nordique. En effet, l'accent porté sur la responsabilisation individuelle fait écho à un rapport officiel sur les réformes de l'État-providence qui appelait à une plus grande implication du citoyen dans la régulation sociale. Il soulignait notamment que : «Les structures peuvent conduire à la passivité et au renforcement des problèmes alors que les individus disposent souvent en eux-mêmes d'une grande part de la solution à leurs propres problèmes ») (SoU, 2003, p. 160). Cette tendance à l'individualisation est également cohérente avec la prise en considération croissante de l'équité au détriment de l'égalité, qui s'est notamment traduit par une diminution des taux marginaux d'imposition sur le revenu.
De surcroît, la dégressivité des allocations chômage et les allégements fiscaux répondent à la volonté de stimuler le développement des emplois peu qualifiés tels que les services à la personne. En effet, les dispositifs de formation professionnelle ne sont pas suffisants pour stimuler l'emploi. Ainsi, l'économie suédoise n'a créé que 30000 emplois en 2006 avec un taux de croissance de 4,2\% alors que le stock de chômeurs est estimé à près de 500000 . Le développement des emplois peu qualifiés et par conséquent moins rémunérés, est une nécessité pour relever le taux d'activité. Il induit toutefois une segmentation du marché du travail qui pose problème au regard des conceptions traditionnelles de l'État-providence (EsPING-ANDERSEN, 2001). Le développement de ces emplois a en effet présidé à l'apparition au Royaume-Uni, des Working Poor qui n'arrivent pas à construire une trajectoire professionnelle ascendante. Or la société suédoise ne pourrait accepter de voir la politique de l'emploi générer durablement des trappes de pauvreté. Les dernières réformes de l'assurance chômage sont ainsi susceptibles de stimuler une réflexion portant sur l'ensemble des politiques sociales afin de dégager les moyens nécessaires pour limiter les effets de la segmentation du marché de l'emploi.

Par ailleurs, l'absence de concertation avec les syndicats lors de la réforme de l'assurance chômage en 2006 est également digne d'être signalée. Il est néanmoins trop tôt pour formuler des conclusions sur l'évolution de la gouvernance du marché du travail en Suède. En effet, l'attitude du nouveau gouvernement peut s'expliquer par la forte orientation politique des syndicats suédois qui soutiennent traditionnellement le parti social-démocrate (JørGENSEN, 2003, p. 135). Or il n'est pas exclu que les syndicats suédois prennent à terme exemple sur la confédération syndicale danoise LO qui a rompu en 2003 son attachement au parti social démocrate afin d'accroître sa marge de manœuvre politique. En effet, les syndicats suédois continuent de repenser les moyens de leur influence consécutivement à la disparition formelle du corporatisme qui imprégnait les institutions en charge de la politique de l'emploi en Suède. 


\section{Bibliographie}

AnXo D., Erhel C. (1998), «La politique de l'emploi en Suède: nature et évolution», in Barbier J.-C. et Gautié J. (ed), Les politiques de l'emploi en Europe et aux ÉtatsUnis, Paris, PUF.

ArbetSlÖSHETSKASSORNAS SAMORGANisATION (2006), Historisk om arbetslöshetsförsäkringen, Stockholm.

ARBETSMARKNADSDEPARTEMENTET, Đirektiv om obligatorisk arbetslöshetsförsäkring, Stockholm, Juni (2007).

BARBIER J.-C. (2000), «À propos des difficultés de traduction des catégories d'analyse des marchés du travail et des politiques de l'emploi en contexte comparatif européen », Centre d'études de l'emploi, document de travail $\mathrm{n}^{\circ} 3$, septembre.

BoISARD P. (2006), «L'aide au retour à l'emploi en Suède», Sociétal no 52 , deuxième trimestre.

Boyer R. (2006), La flexibilité danoise. Quels enseignements pour la France? Paris, éditions Rue d'Ulm.

CALmfors L. (1994), "Active labour market policy and unemployment - A framework for the analysis of crucial design features", OECD Economic Studies $n^{\circ} 22$, Spring.

Castel R. (2005), Les métamorphoses de la question sociale, Paris, Gallimard.

Coulet C. (2005), «Le dilemme du prisonnier résolu: les voies de collaboration entre les partenaires sociaux suédois », Nordiques, $\mathrm{n}^{\circ} 8$.

Edebalke P. (1975), G., Arbetslöshetensförsäkringsdeb atten. En studie I svensk socialpolitik 1892-1934, Lund universitet.

EDLING N. (2005), Om arbetslöshetens problem och introduction av communal arbetsförmedling 1890-1920, Föredrag vid historiskmötet i Uppsala, april.

Erixon L. (2005), Travelling Along the Third Way. A Swedish Model of Stabilisation Equity and Growth, Department of Economics, Stockholm University, Working Paper in Economics 2005 : 10, Stockholm.

ESPING-ANDERSEN G. (2001), «Un État-providence pour le XXI ${ }^{\mathrm{e}}$ siècle» in Daniel C. et Palier B. (eds), La protection sociale en Europe. Le temps des réformes, Paris, La Documentation française.

FInANSDEPARTEMENTET (2006), Svensk aktiveringspolitik i nordiskt perspektiv, Stockholm.

Forslund A., Holmlund B. (2003), Arbetslöshet och arbetsmarknadspolitik, Stockholm, Institut för Arbetsmarknadspolitisk Utvärdering, rapport 2003 : 6 .

Freyssinet J. (2000), «Plein emploi, droit au travail, emploi convenable», Revue de L'IRES no 34, 2000/3.

FURÅKER B. (1977), Stat och arbetsmarknad, Lund universitet.
HJERTNer THORÉn (2005), Kommunal aktiveringspolitik K. : en fallstudie av det praktiska arbetet med arbetslösa socialbidragstagare, Institutet för arbetsmarknadspolitisk utvärdering, working paper 2005 : 11.

Johansson P., Martinson S. (2001), Varför lyckades det nationella IT-programmet, Swit?-en jämförelse mellan två arbetssät, Ekonomisk Debatt, årg 29, no 4.

JøRGENSEN H. (2003), «Le rôle des syndicats dans les réformes sociales en Scandinavie dans les années 1990», Revue française des affaires sociales $\mathrm{n}^{\circ} 4$, octobredécembre.

Kvist J. (2003), «Les stratégies scandinaves d'activation dans les années 1990: vers un remaniement du concept de citoyenneté sociale et du modèle social scandinave», Revue française des affaires sociales $\mathrm{n}^{\mathrm{o}} 4$, octobredécembre.

LindBom T. (1938), Den svenska fackföreningens uppkomst och tidigare historia 1872-1900, Stockholm.

Lundin M. (2005), “Dœs Cooperation Improve Implementation? Central local governement relations in active labour market policy in Sweden", Institutet för arbetsmarknadspolitisk utvärdering, working paper $2005: 2$.

Mansuy M. (2005), «Politiques et institutions danoises d'aide au retour à l'emploi», Paris, les papiers du CERC $\mathrm{n}^{\mathrm{o}} 2$.

MARTin J.-P. (2000), "What works among active labour market policies: evidence from OECD countries'experience" ", OECD Economic Studies no 30, Paris, OCDE.

OlOFSSON J.(1996), Arbetslöshetsfrågan I historisk belysning. En diskussion om arbetslöshet och social politik i Sverige 1830-1920, Lund, Studentlitteratur.

Regeringens Proposition (2006), En arbetslöshetsförsäkring för arbete, Stockholm, 2006/07 : 15.

Rothstein B. (1986), Den socialdemokratiska staten, Arkiv avhandlingsserie 21, Lund Political Studies.

Rothstein B. (1988), "Social Classes and Political Institutions: the Roots of Swedish Corporatism", Maktutredningen, October.

SiANESI B. (2001), "An Evaluation of the Active Labour Market Programmes in Sweden”, Institutet för arbetsmarknadspolitisk utvärdering, Stockholm, working paper $2001: 5$.

SOCIALFÖRSÄKRINGSUTREDNINGEN (2005), Vad är arbetslinjen? Stockholm, Samtal om Socialförsäkring $n^{0} 4$.

Social Security BoArd (1937), Unemployment Compensation. What and Why?, Publication №. 14, Washington, United States Government Printing Office. 
Sou (1936), Svensk arbetslöshetspolitik åren 1914-1935, Stockholm, 1936: 32.

Sou (1944), Statsmakterna och folkhushållningen under den till följd av stormaktsriget 1939 inträdda krisen, Stockholm, 1944: 11.

Sou (1968), Arbetsmarknadsverket och arbetmarknadspolitiken, Stockholm, 1968: 61.

Sou (1974), Att utvärdera arbetsmarknadspolitik, Stockholm, $1974: 29$.

Sou (1999), Avkorporativisering och lobbyism, Stockholm, 1999: 121.
Sou (2000), Välfärld vig vägskäl. Utvecklingen under 1990-talet, Stockholm, $2000: 3$.

Sou (2003), Utvecklingskraft för hållbar välfärd, Stockholm, 2003 : 123.

STATENS ARBETSLÖSHETSKOMMISSION (1929), Det svenska samhället och arbetslösheten 1914-1924, Stockholm.

STATSKONTORET (1999), Vem ser efter arbetlöshetskassorna? Stockholm, 1999: 32. 